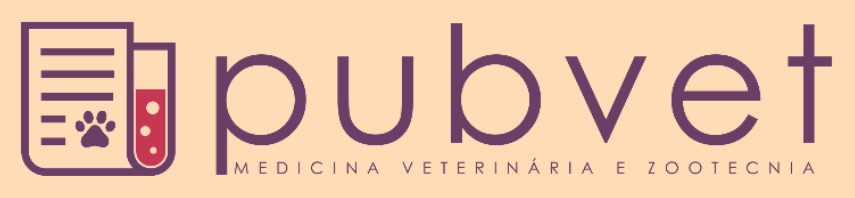

ISSN 1982-1263

https://doi.org/10.31533/pubvet.v13n4a318.1-5

\title{
Escore APGAR: Aplicação em neonatos caninos nascidos de parto normal e cesariana
}

\author{
Ana Gabriela Pereira Moura Leite ${ }^{*} \bullet$, Maria Luíza Lima Cordeiro ${ }^{\bullet}$, Suzane de Cássia Moraes \\ Albuquerque $^{1}$, Francisca Elda Ferreira Dias ${ }^{3}$, Mônica Arrivabene ${ }^{4}$, Tânia Vasconcelos Cavalcante $^{4 * \bullet}$ \\ ${ }^{I}$ Acadêmica de Medicina Veterinária- Programa de Iniciação Científica Voluntária/ICV, Universidade Federal do Piauí. \\ Médico(a) Veterinário(a), Universidade Federal do Piauí, Teresina-Pi, Brasil. \\ ${ }^{3}$ Docente do curso de Medicina Veterinária da Universidade Federal do Tocantins, Araguaína-TO, Brasil-E-mail:eldadias@uft.edu.br \\ ${ }^{4}$ Docente do curso de Medicina Veterinária da Universidade Federal do Piaui, Teresina-Pi, Brasil \\ *Autor para correspondência: gabimouraleite@hotmail.com e cavalcante.tv@gmail.com
}

Resumo. Define-se neonatologia como a ciência responsável pelo estudo dos cuidados aos neonatos, aos seus aspectos fisiológicos e às suas afecções neonatais propriamente ditas. $\mathrm{O}$ sistema de escore Apgar é um método fácil e confiável para avaliar a vitalidade de neonatos animais. No presente trabalho objetivou-se avaliar a vitalidade de neonatos caninos nascidos de parto eutócico e cesariana. Foram coletados dados de 31 neonatos de cães de diferentes portes e raças. Desse número amostral, 21 nasceram de parto eutócico e 10 através de cesariana. Coletaram-se os dados cerca de 10 minutos após o nascimento, aferindo-se: frequências cardíacas, respiratórias e inspecionados a coloração da mucosa gengival, tônus muscular e irritabilidade reflexa, atribuindo-lhes notas de 0 a 2 . A somatória das notas específica de cada variável conferiu aos neonatos o escore Apgar total de 0 a 10. Os resultados foram submetidos à análise de variância ao nível de 5\% de probabilidade e realizou-se o teste de normalidade e homogeneidade. As variáveis foram comparadas pelo teste t- Student, ao nível de 5\% de probabilidade. As frequências cardíaca, respiratória e escore Apgar foram influenciados pelos tipos de parto. As maiores médias de frequências cardíacas, respiratórias e escore Apgar de 184,05 \pm 6,95 bpm e 47,23 $\pm 2,18 \mathrm{mpm}$ e 8,95 \pm 0,22 , respectivamente, foram encontrados em animais nascidos de parto eutócico. O escore Apgar demonstrou-se um método eficiente na avaliação neonatal de cães, bem como colaborou para identificação de alterações inespecíficas do período neonatal imediato.

Palavras chave: assistência pós-natal, caninos, neonatologia

\section{APGAR score: Application in canine neonates born normal and cesarean}

Abstract. Neonatology is defined as the science responsible for the study of neonatal care,
its physiological aspects and its neonatal affections per se. The Apgar score system is an
easy and reliable method to evaluate the vitality of newborn animals. The present study
aimed to evaluate the vitality of canine neonates born from euthy- partal delivery and
cesarean delivery. Data were collected from 31 neonates of dogs of different sizes and
breeds. Of this sample number, 21 were born with eutocic delivery and 10 with caesarean
section. The data were collected about 10 minutes after birth, measuring: heart rate,
respiratory rate, and inspection of the gingival mucosa, muscle tone and reflex irritability,
assigning scores from 0 to 2 . The sum of the notes of each variable gave newborns the total
Apgar score of 0 to 10 . The results were submitted to analysis of variance at the level of
$5 \%$ of probability and the test of normality and homogeneity was performed. The variables
were compared by t-Student test, at a $5 \%$ probability level. Heart rate, respiratory rate and
Apgar score were influenced by the types of delivery. The highest mean heart rate,
respiratory and Apgar scores of $184.05 \pm 6.95$ bpm and $47.23 \pm 2.18$ mpm and $8.95 \pm 0.22$, 
respectively, were found in animals born with euthyococcal labor. The Apgar score proved to be an efficient method in the neonatal evaluation of dogs, as well as collaborated to identify nonspecific alterations of the immediate neonatal period.

Keywords: postnatal care, canines, neonatology

\section{Calificación APGAR: Aplicación en neonatos caninos nacidos de parto normal y cesárea}

Resumen. Se define neonatología como la ciencia responsable del estudio de los cuidados a los recién nacidos, a sus aspectos fisiológicos y a sus afecciones neonatales propiamente dichas. El sistema de puntuación APGAR es un método fácil y confiable para evaluar la vitalidad de los animales recién nacidos. En el presente trabajo se objetivó evaluar la vitalidad de neonatos caninos nacidos de parto eutócico y cesárea. Se recogieron datos de 31 neonatos de perros de diferentes portes y razas. De ese número muestral, 21 nacieron de parto eutócico y 10 a través de cesárea. Se recogieron los datos unos 10 minutos después del nacimiento, midiendo: frecuencia cardíaca, frecuencia respiratoria, e inspeccionados a la coloración de la mucosa gingival, tono muscular e irritabilidad refleja, asignándoles notas de 0 a 2. La suma de las notas de las variables de cada variable conferido a los neonatos el puntaje Apgar total de 0 a 10. Los resultados fueron sometidos al análisis de varianza al nivel del 5\% de probabilidad y se realizó la prueba de normalidad y homogeneidad. Las variables fueron comparadas por la prueba de $t$-Student, al nivel del 5\% de probabilidad. Las frecuencias cardiacas, respiratorias y escasas de Apgar fueron influenciadas por los tipos de parto. Las mayores medias de frecuencia cardiaca, respiratoria y clasificación Apgar de 184,05 $\pm 6,95$ bpm y 47,23 $\pm 2,18 \mathrm{mpm}$ y 8,95 $\pm 0,22$, respectivamente, fueron encontradas en animales nacidos de parto eutócico. El puntaje Apgar se demostró un método eficiente en la evaluación neonatal de perros, así como colaboró para la identificación de alteraciones inespecíficas del período neonatal inmediato.

Palabras clave: asistencia post-natal, caninos, neonatología

\section{Introdução}

Define-se neonatologia como a ciência responsável pelo estudo dos cuidados aos neonatos, aos seus aspectos fisiológicos e às suas afecções neonatais propriamente ditas (Lucio et al., 2009). A neonatologia vem despertando o interesse de grande parte dos médicos veterinários, isso ocorre pelo fato de que até $30 \%$ dos filhotes podem vir a óbito antes do desmame, taxa que se eleva até a puberdade (Prats et al., 2005). Para Sorribas (2007) essa alta taxa de mortalidade, estimada de 20 a 30\%, ocorre devido a imaturidade fisiológica e imunológica dos neonatos, que os tornam extremamente sensíveis ao ambiente, aos agentes infecciosos e parasitários, tornando assim as doenças neonatais um grande desafio ao clinico veterinário. A morte neonatal ocorre mais comumente durante a expulsão, após nascimento, nas primeiras semanas de vida, ou após o desmame. No entanto, a taxa de mortalidade perinatal (cachorros mortos e neonatos) é maior durante o parto, imediatamente após o nascimento e nos primeiros dias de vida (Münnich, 2008). As principais causas de mortalidade neonatal são dificuldades respiratórias após o parto e infecção bacteriana (Concannon et al., 2003).

A inaptidão em proceder ao exame físico detalhado associado à carência do conhecimento técnicocientífico em Neonatologia Veterinária colaboram para o diagnóstico impreciso e o tratamento empírico as afecções e justificam os elevados índices de mortalidade apresentados (Lucio et al., 2009).

O teste de Apgar modificado para cães foi proposto com base nas regras propostas pela médica norte americana doutora Virginia Apgar e utilizadas em bebês. Consta em avaliar cinco sinais objetivos facilmente detectáveis, sem a utilização de ferramentas sofisticadas: frequência cardíaca, esforço respiratório, irritabilidade reflexa e motilidade e coloração das mucosas (Veronesi et al., 2009). Desta forma, objetivou-se avaliar a vitalidade neonatal de cães de parto normal e cesárea, pela aplicação do escore de Apgar. 


\section{Escore APGAR: Aplicação em neonatos caninos}

\section{Material e métodos}

Foram coletados dados de 31 neonatos de cães de diferentes portes e raças, advindo de partos normais e cesarianas. Desse número amostral, 21 nasceram de parto normal e 10 de cesariana. Os dados foram coletados cerca de 10 minutos após o nascimento, sendo aferidas frequências cardíacas e respiratórias e inspecionados a coloração da mucosa gengival, tônus muscular e irritabilidade reflexa, atribuindo-lhes notas de 0 a 2, conforme a tabela 1 . Onde a somatória das notas específicas de cada variável conferiu aos neonatos o escore Apgar total de 0 a 10 . O tônus muscular foi avaliado pela capacidade do neonato em flexionar membros e musculatura abdominal quando em posição ventro-dorsal, enquanto a irritabilidade reflexa foi observada por meio da resposta neonatal à manipulação durante o exame clínico e provocando um estimulo de dor (Figura 1).

Tabela 1. Escore de Apgar modificado para neonatos caninos.

\begin{tabular}{lccc}
\hline Parâmetros avaliados & \multicolumn{3}{c}{ Escore } \\
\cline { 2 - 4 } & 0 & 1 & 2 \\
\hline Frequência Cardíaca & Ausente & $<180 \mathrm{bpm}$ & 180 a 250 bpm \\
Frequência Respiratória & Ausente & $<15 \mathrm{mpm}$ & $>15$ mrm \\
Tônus Muscular & Flacidez & Alguma Flexão & Movimentação Ativa \\
Irritabilidade Reflexa & Ausente & Vocalização & Vocalização alto \\
Coloração da Mucosa & Cianótica & Pálido & Rósea \\
\hline Total & 0 & 5 & 10
\end{tabular}

Fonte: Veronesi et al.(2009)

Os dados foram testados quanto à normalidade e homogeneidade de variâncias pelos testes de Shapiro-Wilk e Bartlett, respectivamente; e, em seguida, submetida à análise de variância (ANOVA) ao nível de 5\% de probabilidade. As variáveis frequências cardíaca, respiratória e escore Apgar foram comparadas nos grupos (parto cesariana e eutócico) pelo teste t-Student, ao nível de 5\% de probabilidade, respectivamente.

Todos os procedimentos realizados neste experimento foram aprovados pela Comissão de Ética no Uso de Animais da Universidade Federal do Piauí (CEUA/UFPI), no Centro de Ciências Agrárias sob número 347/17.
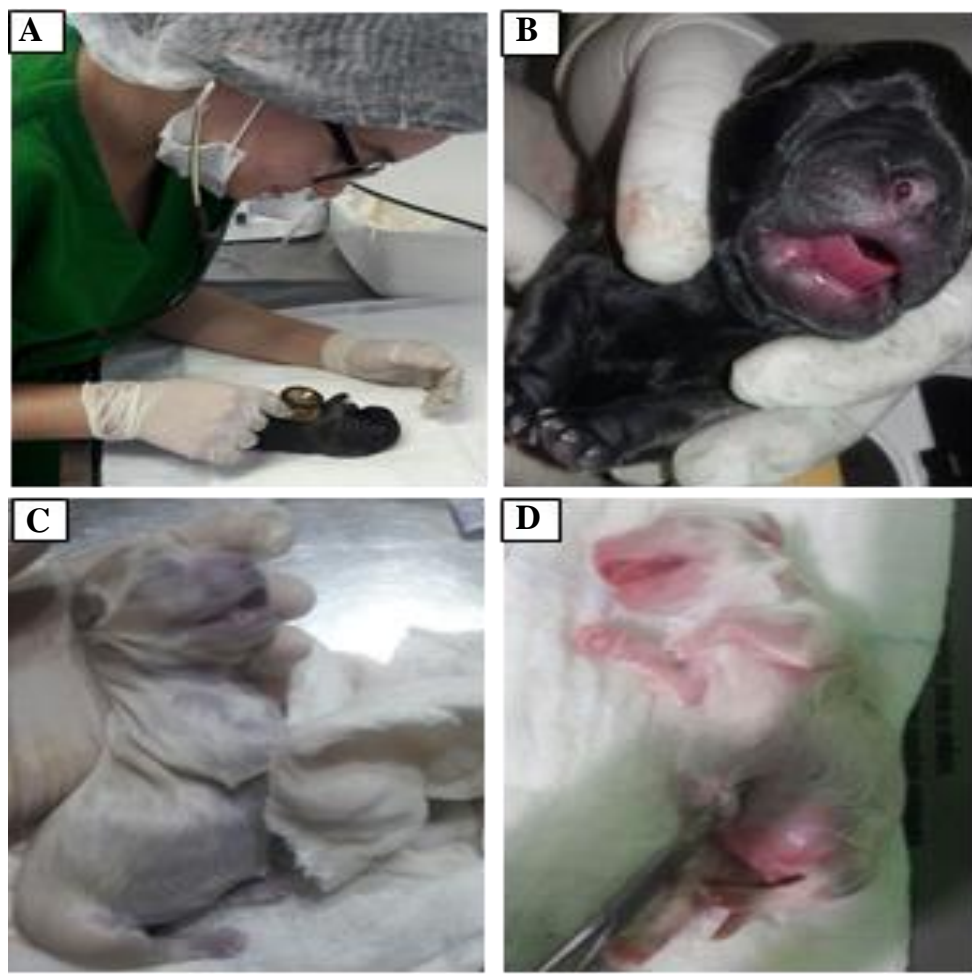

Figura 1. Avaliação da frequência cardíaca (A) e coloração da mucosa (B); neonato cianótico após cesariana $(\mathbf{C})$ e após reanimação $(\mathbf{D})$. 


\section{Resultados e discussão}

A maior média para a frequência cardíaca de $184,0 \pm 6,95 \mathrm{bpm}$ foi observado em animais provenientes de parto eutócico, com incremento expressivo de 13,4\% em relação aos animais nascidos de cesariana (Figura 2A). Esse resultado pode ser justificado devido às alterações cardiocirculatórias da mãe em decorrência da anestesia que foi realizado nas cesarianas (Gabas et al., 2006).

Gabas et al. (2006) avaliando o estado neurológico e cardiorrespiratório de filhotes de cães nascidos de parto normal ou de cesariana sob anestesia geral inalatória com sevofluorano, também verificaram os maiores valores de frequência cardíaca cerca de $195 \mathrm{bpm}$ em cães nascidos de parto eutócico. Já Lucio et al. (2009) não relataram diferença de frequência cardíaca aos 5 minutos após o nascimento entre os dois tipos de parto.

A média da frequência cardíaca dos neonatos nascidos por parto eutócico, apesar de terem sido maiores que o grupo por cesariana, está abaixo do valor preconizado por Barreto (2003) e Peixoto \& Bezerra Junior (2010). De acordo com os autores a frequência cardíaca esperada em filhotes de cães é superior a $200 \mathrm{bpm}$. A perfusão periférica do neonato é mantida devido a sua frequência cardíaca elevada, sendo esta a única maneira de manter a pressão sanguínea, por outro lado apresenta pressão venosa elevada, onde desta forma a circulação neonatal é considerada como um sistema de baixa resistência e alto fluxo, diferentemente do adulto, o que minimiza a carga de trabalho cardíaco (Moon et al., 2001).

A frequência respiratória avaliada nos dois grupos demonstrou estar dentro do intervalo de normalidade (15-40 mpm). A maior média de 47,23 $\pm 2,18 \mathrm{mpm}$ foi encontrada em animais oriundos de parto Eutócico (Figura 2B). Tal fato pode ser atribuído à depleção respiratória em cesarianas em razão da exposição fetal prolongada aos anestésicos (Gaido, 1997).

Em ambos os grupos, observou-se que as frequências respiratórias foram superiores às obtidas por Barreto (2003) e Peixoto \& Bezerra Junior (2010) de 15 a $35 \mathrm{mpm}$ em neonatos caninos. Entretanto, no período fetal, a hipóxia causa redução nos movimentos respiratórios e falta de estímulo respiratório e, no pós-parto, os neonatos respondem de forma semelhante ao período fetal, com diminuição da frequência respiratória frente à elevação da pressão parcial de dióxido de carbono $\left(\mathrm{CO}_{2}\right)$ e à diminuição de oxigênio, contrariando os resultados deste trabalho. Já para Landim-Alvarenga et al. (2006) a resposta ventilatória neonatal à hipóxia é bifásica, com elevação inicial da frequência respiratória seguida por um declínio progressivo. Os autores comentam ainda que as estimulações tátil e térmica das regiões genital ou umbilical induzem ao reflexo respiratório nos primeiros três dias após o nascimento.

A avaliação da vitalidade neonatal de cães pelo escore Apgar apresentou superioridade em neonatos nascidos de parto eutócico $(8,95 \pm 0,22)$ em relação aos nascidos por cesariana $(7,20 \pm 0,22)$ (Figura 2C). Gabas et al. (2006) citam que a pontuação da coloração da mucosa nos neonatos nascidos de cesariana no primeiro minuto de vida, é menor em relação às dos filhotes nascidos por parto eutócico, e isso, deve-se à apneia logo após o nascimento, provavelmente, em razão da depressão neurológica causada pelos agentes de manutenção anestésica.
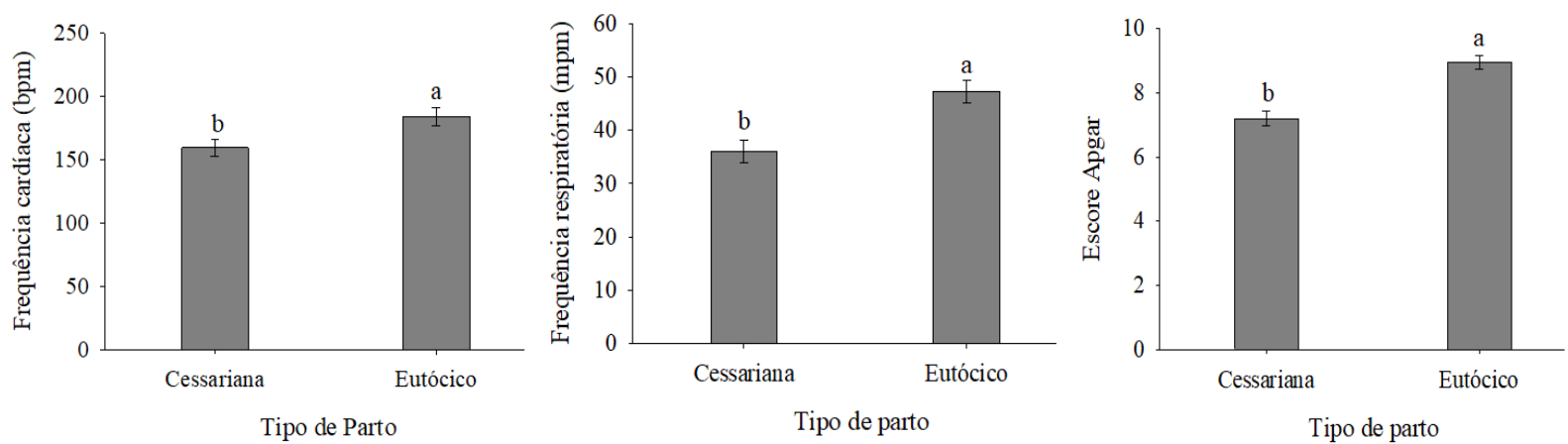

Figura 2. Frequência cardíaca (A), Frequência respiratória (B) e Escore Apgar (C) de neonatos caninos avaliados logo após o nascimento. Teresina-PI, 2017. Médias seguidas de letras distintas, diferem entre si pelo teste t de Student ao nível de $5 \%$ de probabilidade. 


\section{Escore APGAR: Aplicação em neonatos caninos}

Segundo Traas (2008) escore de Apgar com valores 8 a 10 são considerados ideais; 6 a 7 indicam provável asfixia, e que o neonato requer maior atenção, e valores inferiores a 7 são considerados muito baixos (Tabela 1). Os neonatos hígidos devem apresentar mucosas de coloração rósea intenso, sendo assim quando cianóticas, sinalizam severa hipoxemia. E neonatos provenientes de cesariana apresentam depressão respiratória e discreto estímulo à respiração reflexa. Deste modo, a troca gasosa ocorrida durante as primeiras inspirações é menos eficiente e acarreta severa hipóxia e cianose central (Silva, 2008; Spreng, 2004). Silva (2008) destacam que as variáveis tônus muscular e irritabilidade reflexa exprimem o grau de depressão do sistema nervoso central, e que os neonatos nascidos de parto eutócico demonstram excelente evolução destas variáveis, com notas máximas para maioria dos filhotes.

\section{Conclusão}

O escore Apgar é um método de diagnóstico eficiente, tendo em vista que é possível constatar que animais nascidos por parto eutócico apresentam maior viabilidade do que aqueles que nascem pelo parto cesariana. Desta forma, o escore Apgar pode ser utilizado como meio de triagem na rotina clínica neonatal afim de detectar alterações tais como depressão, alterações respiratórias e circulatórias.

\section{Referências bibliográficas}

Barreto, C. S. (2003). Avaliação de filhotes caninos. PhD, Universidade de São Paulo, São Paulo, Brasil.

Concannon, P. W., England, G., III.J., V. \& Linde-Forsberg. (2003). Recent Advaces in Small Animal Reproduction. New York, USA.

Gabas, D. T., Matsubara, L. M., Oliva, V. N. L. d. S., Rodello, L., Rossi, C. N. \& Perri, S. H. V. (2006). Estado neurológico e cardiorrespiratório de filhotes de cães nascidos de parto normal ou de cesariana sob anestesia geral inalatória com sevofluorano. Ciência Rural, 36(5):1450-1455.

Gaido, S. R. (1997). A gestação e a anestesia. Encontro de Anestesiologia Veterinária, 310-16.

Landim-Alvarenga, F. C., Prestes, N. C. \& Santos, T. C. M. (2006). Manejo do neonato. Obstetrícia veterinária. Rio de Janeiro, Brasil: Guanabara Koogan.

Lucio, C. F., Silva, L. C. G., Rodrigues, J. A., Veiga, G. A. L. \& Vannucchi, C. I. (2009). Acid-base changes in canine neonates following normal birth or dystocia. Reproduction in Domestic Animals, 44208-210.

Moon, P. F., Massat, B. J. \& Pascoe, P. J. (2001). Neonatal ritic care. Veterinary Clinics of North America: Small Animal Practice, 31(2):343-367.

Münnich, A. (2008). The pathological newborn in small animals: the neonate is not a small adult. Veterinary Research Communications, 32(1):81-85.

Peixoto, G. C. X. \& Bezerra Junior, R. Q. (2010). Cuidados básicos com o neonato canino: uma revisão. PUBVET, 4717-722.

Prats, A., Prats, A., García, F., Dumon, C., Martí, S. \& Coll, V. (2005). Neonatologia e pediatria canina e felina: Interbook.

Silva, L. C. G. (2008). Parâmetros clínicos, hemogasométricos e radiográficos para avaliação respiratória de neonatos caninos nascidos em eutocia ou cesariana eletiva. $\mathrm{PhD}$, Universidade de São Paulo, São Paulo, Brasil.

Sorribas, C. E. (2007). Atlas de neonatologia y pediatria en caninos: Inter-medica.

Spreng, D. (2004). Intensive care of neonates and pediatrics. Paper presented at the 29th World Congress of the Word Small Animal Veterinary Association, Rhodes.

Traas, A. M. (2008). Resuscitation of canine and feline neonates. Theriogenology, 70(3):343-348.

Veronesi, M. C., Panzani, S., Faustini, M. \& Rota, A. (2009). An Apgar scoring system for routine assessment of newborn puppy viability and short-term survival prognosis. Theriogenology, 72(3):401-407.

Recebido: 19 de março, 2019.

Aprovado: 18 de abril, 2019

Publicado: 7 de maio, 2019.

Licenciamento: Este artigo é publicado na modalidade Acesso Aberto sob a licença Creative Commons Atribuição 4.0 (CC-BY 4.0), a qual permite uso irrestrito, distribuição, reprodução em qualquer meio, desde que o autor e a fonte sejam devidamente creditados. 